\title{
Procesos de determinación del derecho a la salud bucal de mujeres en post-parto y sus hijos. Bucaramanga y Medellín (Colombia), Santiago (Chile), 2017*
}

\section{Determination processes of the right to oral health of postpartum women and their children. Bucaramanga and Medellín (Colombia), Santiago (Chile), 2017}

\section{Processos de determinação do direito à saúde bucal de mulheres em pós-parto e seus filhos. Bucaramanga e Medellín (Colômbia), Santiago (Chile), 2017}

Recibido: 05 de noviembre de 2019. Aceptado: 10 de noviembre de 2020. Publicado: 30 de junio de 2021.

DOI: https://doi.org/10.11144/Javeriana.rgps20.pdds

\author{
Ángela María Franco Cortés \\ Universidad de Antioquia, Colombia \\ ORCID: https://orcid.org/0000-0001-8579-058X \\ Marco Antonio Cornejo Ovalle \\ Universidad de Chile, Chile \\ ORCID: https://orcid.org/0000-0001-9147-5754 \\ Iris Lucía Espinoza Santander \\ Universidad de Chile, Chile \\ ORCID: https://orcid.org/0000-0001-8949-8885 \\ Clara Liliana Gallego Gómez \\ Universidad Cooperativa de Colombia, Colombia \\ ORCID: https://orcid.org/0000-0001-9317-551X \\ Valentina de la Roche Colorado ${ }^{a}$ \\ Universidad de Antioquia, Colombia \\ ORCID: https://orcid.org/0000-0002-2827-7277 \\ Valentina Fajreldin Chuaqui \\ Universidad de Chile, Chile \\ ORCID: https://orcid.org/0000-0002-5898-3906 \\ Oriana Delgado Restrepo \\ Universidad de Antioquia, Colombia \\ ORCID: https://orcid.org/0000-0002-0265-7183 \\ Andrea Almario Barrera \\ Universidad Santo Tomás, Colombia \\ ORCID: https://orcid.org/0000-0002-8020-4091 \\ Sonia Constanza Concha Sánchez \\ Universidad Santo Tomas, Colombia \\ ORCID: https://orcid.org/0000-0003-3092-520X
}

Para citar este artículo: Franco AM, Cornejo MA, Espinoza IL, Gallego CL, de la Roche V, Fajreldin V, Delgado O, Almario A, Concha S. Procesos de determinación del derecho a la salud bucal de mujeres en postparto y sus hijos. Bucaramanga y Medellín (Colombia), Santiago (Chile), 2017. Rev Gerenc Polit Salud. 2021;20. https://doi.org/10.11144/Javeriana.rgps20.pdds

${ }^{*}$ Artículo de investigación.

${ }^{\text {a }}$ Autora de correspondencia. Correo electrónico: valentinadelarochec@gmail.com 


\section{Resumen}

Introducción. Numerosos estudios abordan la situación de salud bucal y el acceso a la atención odontológica de las gestantes, sin embargo pocos continúan la observación en el postparto y mucho menos la situación de sus hijos durante los primeros años de vida. Objetivo. Identificar y comprender los procesos generales, particulares y singulares que limitan o favorecen el ejercicio del derecho a la salud bucal de la mujer postparto y su hijo durante el primer año de vida. Método. El estudio parte del enfoque de la Determinación Social de la Salud en dos ciudades colombianas, Medellín y Bucaramanga, y en Santiago de Chile. En cada ciudad participaron 26 binomios previo consentimiento informado. Se usaron técnicas cualitativas (entrevistas y grupos de discusión) y cuantitativas (encuesta y exámenes bucales) para la recolección de los datos. Resultados. Procesos generales de orden económico, político, y de género, unidos a dinámicas laborales, de consumo y socioculturales, de los modos de vida de las familias, determinan, positiva o negativamente, las oportunidades de acceso al cuidado de la salud bucal para ambos sujetos y el estado objetivo de la misma. Conclusión. Los procesos de nivel general, con algunas diferencias entre los dos países, limitan el ejercicio del derecho a la salud reconocido como fundamental por ambas constituciones políticas. Esos procesos del nivel general, subsumen los procesos particulares y singulares que también se comportan como limitantes en la mayoría de los casos.

Palabras clave: Derecho a la salud, salud bucal, mujeres, periodo postparto, lactante.

\section{Abstract}

Introduction. Numerous studies address the oral health situation and access to dental care for pregnant women, however few maintain postpartum observation, much less the situation of the children during their first years of life. Objective. To identify and understand the general, particular and singular processes that limit or favor the exercise of the right to oral health of postpartum women and their children during the first year of life. Method. The study utilizes the Social Determinants of Health approach in two Colombian cities, Medellín and Bucaramanga, and in Santiago de Chile. In each city, 26 pairs participated, with prior informed consent. Qualitative techniques (interviews and discussion groups) and quantitative techniques (survey and oral exams) were used for data collection. Results. General economic, political, and gender processes, together with labor, consumer, and sociocultural dynamics of the families' lifestyles, determine, positively or negatively, the opportunities of access to oral health care for both subjects and for their objective condition. Conclusion. Processes at a general level, with some differences between the two countries, limit the exercise of the right to health, recognized as fundamental by both political constitutions. Those processes at the general level subsume the particular and singular processes that also behave as limitations in most cases.

Keywords: Right to health, oral health, women, postpartum period, breastfed baby.

\section{Resumo}

Introdução. Numerosos estudos abordam a situação de saúde bucal e o acesso à atenção odontológica das gestantes, porém poucos continuam a observação no pós-parto e ainda menos a situação de seus filhos durante os primeiros anos de vida. Objetivo. Identificar e compreender os processos generais, particulares e singulares que limitam ou favorecem o exercício do direito à saúde bucal da mulher pós-parto e seu filho durante o primeiro ano de vida. Método: $\mathrm{O}$ estúdio parte do enfoque da Determinação Social da saúde, em duas cidades colombianas (Medellín y Bucaramanga), e em Santiago de Chile. Em cada cidade participaram 26 binômios com consentimento prévio informado. Usaram-se técnicas qualitativas (entrevistas e grupos de discussão) e quantitativas (pesquisas e exames bucais) para a coleta de dados. Resultados. As oportunidades de aceso ao cuidado da saúde bucal para ambos sujeitos e o estado objetivo de tal atenção estão determinados, positiva ou negativamente, por processos de nível geral de ordem económica, política e de género. A estes fatores deve-se acrescentar as dinâmicas laborais, de consumo e socioculturais, bem como os modos de vida das famílias. Conclusão. Os processos de nível geral, com algumas diferenças entre os dois países, limitam o exercício do direito à saúde, reconhecido como fundamental por ambas as duas constituições políticas. Esses processos de nível geral subsomem os processos particulares e singulares que também agem como limitantes na maioria dos casos.

Palavras-chave: Direito à saúde, saúde bucal, mulheres, período pós-parto, Direito, lactante. 
Procesos de determinación del derecho a la salud bucal de mujeres en post-

parto y sus hijos. Bucaramanga y Medellín (Colombia), Santiago (Chile), 2017

\section{Introducción}

Las mujeres y los niños ${ }^{1}$ cursan etapas de la vida en las que la salud bucal cobra mayor importancia por su relación con algunos procesos vitales. Durante la gestación las mujeres necesitan una buena salud bucal para tener una adecuada alimentación que garantice los nutrientes necesarios para ella y para el desarrollo del feto. Los niños recién nacidos y durante los primeros años de vida necesitan de la salud bucal para los procesos biológicos que garantizan un crecimiento y desarrollo adecuado, más cuando en esta etapa la boca es el vehículo a través del cual establecen los lazos afectivos con su madre y otros miembros de la familia y con el cual conocen el mundo que les rodea.

La cobertura de la atención odontológica para las mujeres gestantes en algunas regiones o países alcanza niveles inferiores al 20\%, mientras en otros cubre a más del $80 \%$ de las embarazadas (1). Las investigaciones también señalan que las más afectadas son las mujeres gestantes de los grupos sociales más desfavorecidos, las residentes en las zonas rurales, las vinculadas a los sistemas públicos de atención o aquellas que están por fuera de estos sistemas (2-5).

El interés por la salud bucal de las mujeres durante el periodo de postparto es menor que en el embarazo. En el ámbito académico son pocas las investigaciones que abordan este tema $(2,6,7)$ $\mathrm{y}$, por lo general, analizan el impacto que la condición bucal de la madre puede tener sobre la de su hijo (8) y los resultados de las acciones promocionales, preventivas y educativas para minimizar el riesgo de contaminación vertical por microorganismos cariogénicos (9).

Son pocos los estudios sobre la cobertura de atención odontológica en el postparto (6,7). Y a diferencia de lo que se observa en las políticas y guías de atención en las que se prioriza la atención de las mujeres gestantes no existen lineamientos de política pública ni una normatividad que proteja la salud bucal de las mujeres durante el postparto, lo que sugiere que el derecho a la salud y a su componente bucal no parece ser una prioridad en esta etapa de la vida de la mujer.

En Colombia y en Chile el derecho a la salud está contemplado en la Carta Constitucional y en la práctica este derecho se realiza a través de prestaciones que han ingresado a las leyes de oferta y demanda bajo el supuesto rol rector de un aparato estatal cuyos límites de responsabilidad sobre el sistema de salud son cada vez menores, en coherencia con la teoría neoliberal que propone que el Estado tenga una responsabilidad limitada y se haga cargo sólo de lo que está definido como lo "público" en materia de salud, o sea, de los problemas que suponen altas externalidades y del subsidio de los servicios para la población en extrema pobreza (10).

La Constitución Política colombiana de 1991 consagró que la mujer durante el embarazo y posparto gozará de especial asistencia y protección del Estado (11), en este marco normativo el control prenatal incluye detección temprana de alteraciones del embarazo. Desde 2007 se aprobó la Política Pública Nacional de Primera Infancia "Colombia por la Primera Infancia" donde se estipula que el derecho a la salud del niño comienza con el acto de brindar a la madre y a la pareja la asesoría necesaria en la preparación para la gestación. 
Ángela María Franco Cortés/ Marco Antonio Cornejo Ovalle/ Iris

Lucía Espinoza Santander/ Clara Liliana Gallego Gómez/ et al.

En Chile, la Ley 20.379 de 2009 creó el Sistema de Protección Social "Chile Crece Contigo" que articula diferentes iniciativas sociales y programas de salud y educación con el objetivo de acompañar y hacer seguimiento personalizado a la trayectoria del desarrollo de los niños que se atienden en el sistema público (el 75\%), desde el primer control de gestación hasta su ingreso al sistema escolar (12).

Este estudio reconoce la salud como un proceso histórico social y considera que la posibilidad de ejercer el derecho a la salud es producto de una responsabilidad compartida y de una construcción colectiva, dado que la producción de la salud está determinada por procesos que tienen que ver con lo singular de cada sujeto, con lo particular del grupo social al que pertenece y con lo general de la manera como estructuralmente se organiza la sociedad, lo que significa que su construcción implica trabajar en múltiples propósitos, más allá de contar con un sistema de atención a la enfermedad como tradicionalmente se concibe $(13,14)$.

Pocos estudios, entre ellos los de Gallego y Franco (15) en 2017, Quintero et al. (16) en 2014, Concha y Morales (5) en 2015 y Concha (17) en 2013, llevados a cabo en diversos grupos poblacionales enfatizan en la importancia de reconocer los determinantes del nivel general que incluyen los procesos económicos, políticos y sociales, como elementos que subsumen las dinámica del nivel particular y singular. En estos trabajos también se plantea la necesidad de superar la visión de los modelos tradicionales que se concentran en relacionar las características sociodemográficas de los individuos y del sistema de atención en salud, con el estado de la salud bucal.

Esta investigación buscó comprender cuáles son los procesos generales, particulares y singulares que favorecen y desfavorecen la posibilidad de realización del derecho a la salud bucal de mujeres en periodo de postparto y sus hijos en el primer año de vida, de las ciudades de Bucaramanga y Medellín (Colombia) y Santiago de Chile (Chile), desde el marco interpretativo de la Determinación Social de la Salud, propuesto por la epidemiología crítica (13-14,18-21).

\section{Materiales y métodos}

Se realizó un estudio cualitativo y cuantitativo con enfoque critico-social en tres dimensiones de análisis: el nivel general, que contiene los procesos estructurales que sitúan la salud dentro del ámbito contextual de las políticas socioeconómicas de ambos países; un nivel particular, en el que se identifican los modos de vida de los grupos sociales de los que hacen parte los participantes y su relación con las oportunidades y limitaciones para ejercer el derecho; y el nivel singular que permitió identificar la condición de salud bucal de las personas.

Para la recolección de la información en la dimensión general se realizó un análisis exhaustivo de información estadística y referencial del contexto político, económico y de género de ambos países. Para la dimensión particular se realizó una encuesta que permitió hacer la clasificación de 
la posición social y caracterizar los modos de vida de las 26 familias participantes. A las mujeres se les hizo una entrevista individual semiestructurada y se realizaron grupos de discusión, con lo que se pretendía configurar el "campo de significados" y las nociones y percepciones socioculturales subyacentes al fenómeno de interés (22).

Finalmente, para la dimensión singular se realizaron exámenes odontológicos a la madre y al hijo/a, con base en los criterios del sistema ICDAS (International Caries Detection and Assessment System) modificado (no se tuvo en cuenta condición de cambio visual en el esmalte con superficie seca, código 1). En la madre se evaluó presencia de prótesis y necesidad de elaboración de la misma y además se calculó el índice INTPC (Índice de necesidad de tratamiento periodontal de la comunidad) para la condición periodontal. En el niño se evaluó presencia o antecedente de labio y/o paladar hendido y condiciones de la mucosa bucal.

Se invitaron a participar mujeres de todas las edades, primíparas o multíparas, que se encontraban en el primer trimestre postparto, con sus hijos recién nacidos, residentes en la zona urbana o suburbana de las ciudades mencionadas. Entre las tres ciudades participaron 26 binomios madre/hijo, usuarios del Programa Familia, Mujer e Infancia (FAMI) del Instituto Colombiano de Bienestar Familiar en Medellín y Bucaramanga, o del Programa Chile Crece Contigo en Santiago.

El estudio se realizó en varias etapas:

1. Una vez contactadas e informadas las madres se obtuvo consentimiento escrito sobre su participación. Se realizaron entrevistas en profundidad, cada una de las cuales requirió hasta tres encuentros.

2. Se realizaron uno a dos grupos de discusión por ciudad. Con mujeres diferentes a las entrevistadas, pero que participaban de los mismos programas de gestantes.

3. Una vez terminadas las entrevistas y los grupos de discusión cada mujer participante respondió la encuesta de posición social y modos de vida.

4. Se hizo el examen bucal a la madre y el hijo, al inicio y al cierre de la investigación en un lapso de 12 meses.

La información cualitativa se codificó, tomando como base las categorías establecidas y aquellas emergentes. Después se buscaron los patrones de regularidad de lo expresado (tendencias) y se elaboraron mapas conceptuales para organizar la información por categorías de análisis provenientes de los ítems utilizados en las pautas de entrevista y grupos de discusión. El análisis final de los datos cualitativos condujo a la elaboración de una matriz de procesos críticos (procesos generativos de producción y reproducción social).

Una vez recolectada la información mediante técnicas cuantitativas se sistematizó en hojas de cálculo de Excel y se exportó al paquete estadístico STATA 14 para el procesamiento de los datos. El plan de análisis implicó el cálculo de medidas de resumen según la naturaleza de las variables. 
Ángela María Franco Cortés/ Marco Antonio Cornejo Ovalle/ Iris

Lucía Espinoza Santander/ Clara Liliana Gallego Gómez/ et al.

Después del análisis independiente de los resultados obtenidos bajo técnicas cuantitativas y cualitativas se procedió a realizar triangulación de los mismos buscando contrastarlos, identificar diferencias y similitudes; intentando establecer la convergencia y complementariedad.

\section{Resultados}

Dada la extensión y la riqueza de los datos obtenidos y teniendo en cuenta las tres dimensiones contempladas: general, particular y singular, en la tabla 1 se resume el marco de interpretación de los procesos críticos que interactúan limitando o protegiendo el ejercicio del derecho a la salud bucal de mujeres en periodo de postparto y sus hijos recién nacidos. 
Procesos de determinación del derecho a la salud bucal de mujeres en post-

parto y sus hijos. Bucaramanga y Medellín (Colombia), Santiago (Chile), 2017

Tabla 1. Marco de interpretación de procesos críticos

\begin{tabular}{|c|c|c|c|c|c|}
\hline \multirow{2}{*}{ Dominios } & \multirow{2}{*}{ Dimensiones } & \multicolumn{2}{|c|}{ Procesos críticos } & \multicolumn{2}{|c|}{ Proceso generativos } \\
\hline & & Limitantes & Protectores & Limitantes & Protectores \\
\hline \multirow{3}{*}{ General } & $\begin{array}{l}\text { Económico- } \\
\text { social }\end{array}$ & $\begin{array}{l}\text { Distribución } \\
\text { inequitativa de riqueza, } \\
\text { tierra e ingreso. } \\
\text { Pobreza, desempleo, } \\
\text { informalidad. }\end{array}$ & $\begin{array}{l}\text { Políticas de inversión } \\
\text { social focalizadas en } \\
\text { los estratos } \\
\text { vulnerables, } \\
\text { principalmente en } \\
\text { Chile. }\end{array}$ & & \\
\hline & Política & $\begin{array}{l}\text { Reducción intervención } \\
\text { del Estado, gasto } \\
\text { público e inversión } \\
\text { social. } \\
\text { Políticas focalizadas. } \\
\text { Desregulación y } \\
\text { flexibilización laboral. } \\
\text { Reforma del Sistema de } \\
\text { Salud (regulado por } \\
\text { leyes del mercado). }\end{array}$ & $\begin{array}{l}\text { Chile: antecedente } \\
\text { gobiernos con énfasis } \\
\text { social, Programa Chile } \\
\text { Crece Contigo y } \\
\text { Extensión e } \\
\text { incorporación del } \\
\text { permiso postnatal. } \\
\text { Colombia: políticas de } \\
\text { protección a gestante y } \\
\text { a primera infancia: De } \\
\text { Cero a Siempre, Plan } \\
\text { Nacional de Salud } \\
\text { Pública 2012-2021 }\end{array}$ & & \\
\hline & Género & $\begin{array}{l}\text { Tasa escolaridad y } \\
\text { empleo masculino más } \\
\text { alto. Violencia. } \\
\text { Discriminación. }\end{array}$ & & & \\
\hline Particular & $\begin{array}{l}\text { Dinámicas } \\
\text { socio } \\
\text { culturales }\end{array}$ & $\begin{array}{l}\text { Miedo a odontología. } \\
\text { Idea de que la salud } \\
\text { bucal se ve afectada en } \\
\text { el embarazo. } \\
\text { El cuidado como } \\
\text { asunto meramente } \\
\text { higiénico. } \\
\text { Miedo de las madres } \\
\text { por falta de destreza en } \\
\text { acciones de cuidado. } \\
\text { Convicción de madres, } \\
\text { reforzada por } \\
\text { profesionales, de que el } \\
\text { niño sólo necesita } \\
\text { cuidados en dentición } \\
\text { completa. }\end{array}$ & $\begin{array}{l}\text { Experiencias } \\
\text { educativas sobre } \\
\text { cuidado desde niñez. } \\
\text { Acceso a programas de } \\
\text { atención a gestante y } \\
\text { recién nacido, propicia } \\
\text { empoderamiento, } \\
\text { aprendizajes en } \\
\text { derechos. } \\
\text { Conformación de redes } \\
\text { de apoyo. }\end{array}$ & & \\
\hline Singular & $\begin{array}{l}\text { Estado salud } \\
\text { bucal }\end{array}$ & & & $\begin{array}{l}\text { Madres: alta } \\
\text { experiencia de } \\
\text { caries, necesidad } \\
\text { atención } \\
\text { acumulada. } \\
\text { Primera consulta } \\
\text { tardía hijos }\end{array}$ & $\begin{array}{l}\text { Ninguna } \\
\text { experiencia de } \\
\text { caries niños. } \\
\text { Conductas } \\
\text { preventivas de } \\
\text { madres. }\end{array}$ \\
\hline
\end{tabular}

Fuente: elaborado con base en Breilh (13).

Procesos generales (estructurales) que determinan la realización del derecho a la salud bucal

En términos de los procesos generales, y a pesar de una general similitud en la orientación neoliberal de las políticas económicas y sociales, Chile y Colombia, no avanzan en el modelo 
Ángela María Franco Cortés/ Marco Antonio Cornejo Ovalle/ Iris

Lucía Espinoza Santander/ Clara Liliana Gallego Gómez/ et al.

de desarrollo de la misma manera. La revisión documental da cuenta de un conjunto de factores y procesos cuya dinámica social e histórica es específica en el contexto de cada país.

Más del 35\% de la población colombiana se encuentra en situación de pobreza (23) y para el año 2016 el coeficiente Gini de desigualdad de ingreso fue de 0,51 (24). En Chile este coeficiente, para 2015, fue de 0,49 con tendencia a la baja, aunque un rasgo central de la desigualdad de este país es la concentración del ingreso y la riqueza en el 1\% más rico y según la Organización para la Cooperación y el Desarrollo Económico (OCDE) es uno de los países latinoamericanos con mayor desigualdad $(25,26)$.

En ambos países existe desigualdad en la distribución de la propiedad de la tierra. En Colombia, mientras el $0,06 \%$ de la población posee el $53,6 \%$ de las tierras registradas en el catastro, el $55,6 \%$ posee el 1,7\% (27). En Chile el 70\% de la tierra está distribuida en propiedades que tienen en promedio 14.000 hectáreas, mientras que el $75 \%$ de los propietarios tiene menos del $4 \%$ de la tierra y no alcanzan a tener cinco hectáreas en promedio (28), y el predominio económico de ciertos conglomerados y monopolios económicos se encuentra asociado a familias poderosas $(29,30)$. En Chile, a pesar de los avances económicos persisten las deudas en materia de desigualdad en la distribución de los ingresos, de la propiedad y la riqueza.

En Colombia, las políticas de protección social se construyen sobre estrategias de focalización que se aplican desde hace más de 15 años, a través de programas como "Familias en Acción", "De Cero a Siempre" y otros programas asistenciales que, si bien tienen alguna funcionalidad y han tenido resultados en sectores específicos, con gran impacto político para el manejo del consenso, también han mostrado sus limitaciones como herramienta para erradicar la pobreza y promover la equidad (31). Chile, dado el modelo de desarrollo económico-político que caracterizó a algunos gobiernos postdictadura como una "economía de mercado con fuerte énfasis social" ha incidido sobre algunos indicadores y algunas políticas de inversión social focalizadas en los estratos más vulnerables (32). El avance en la protección de los derechos de los niños se evidencia en la Política Nacional de la Niñez 2015-2025. Se destaca el fortalecimiento a la maternidad, extensión del postnatal para las madres e incorporación del permiso postnatal para padres (Ley 20.545 de 2011).

Las políticas colombianas relativas al sistema de atención en salud se rigen por la Ley 100 de 1993, soportada en la concurrencia plural, pública y privada de aseguradores y prestadores de servicios que compiten en el mercado bajo las reglas de un Estado incapaz y poco interesado en controlar el poder desmedido de las aseguradoras (33). En Chile, en el gobierno de Pinochet se modificó la estructura y el financiamiento del sistema de atención en salud. Se terminó con el Servicio Nacional de Salud, se hizo redefinición de las funciones del Ministerio de Salud (MINSAL) y del Fondo Nacional de Salud (FONASA) y se crearon las Instituciones de Salud Previsional (ISAPRE). En 2004 comenzó la implementación de la reforma que estableció un plan de salud obligatorio para el FONASA y las ISAPRES que incluyen diagnóstico y 
tratamiento estandarizados para un conjunto de enfermedades priorizadas por su alto impacto sanitario y social (34).

Respecto a procesos relacionados con género, en Colombia, a pesar de contar con la Ley 1257 de 2008 (35) de sensibilización, prevención y sanción a formas de violencia contra la mujer, con una Política Pública para la Equidad de Género para las Mujeres y con la Ley 1719 de 2014 por la cual se adoptan medidas para garantizar el acceso a la justicia de las víctimas de violencia sexual, persiste la asimetría histórica en contra de ellas. Las denuncias frente a esta violencia se estrellan con la impunidad y la indiferencia estatal y de la sociedad (36). El hecho de que Michelle Bachelet fuera la primera mujer en alcanzar la presidencia en Chile significó un desafió a las viejas formas de autoridad aportando a la legitimización de las mujeres en los espacios de poder y liderazgo (37). Con todo, las mujeres viven diversas formas de violencia por parte de sus parejas o de su entorno, formas de maltrato que van desde el control, hasta la agresión física y que en la cultura chilena se justifica por el derecho que tienen los hombres sobres las mujeres (38).

Procesos particulares (modos de vida) que determinan la realización del derecho a la salud bucal

La encuesta de identificación de posición social de las mujeres y sus hijos arrojó resultados indicando que la mayoría pertenecían a grupos sociales subasalariados, obreros y en pocos casos de pequeños comerciantes informales. Las madres tenían edades que oscilaban entre 18 y 45 años y residían con sus hijos en asentamientos o barrios precarios (viviendas con carencias, que las cataloga en "condiciones económicas comprometidas"). La afiliación a la seguridad social en salud estaba repartida entre el régimen contributivo y el subsidiado. Solo dos mujeres no estaban afiliadas. En la tabla 2 se describen las principales características de la posición social $\mathrm{y}$ modos de vida de las mujeres encuestadas. 


\section{Ángela María Franco Cortés/ Marco Antonio Cornejo Ovalle/ Iris Lucía Espinoza Santander/Clara Liliana Gallego Gómez/ et al.}

Tabla 2. Aspectos relacionados con la posición social y los modos de vida de las mujeres y sus hijos en Bucaramanga, Medellín y Santiago de Chile

\begin{tabular}{|c|c|c|c|c|c|}
\hline Variable & $\begin{array}{l}\text { Medellín } \\
\text { (n) }\end{array}$ & $\begin{array}{l}\text { Bucaramanga } \\
\text { (n) }\end{array}$ & $\begin{array}{l}\text { Santiago } \\
\text { Chile (n) }\end{array}$ & Total & $\mathbf{P} \uparrow$ \\
\hline \multicolumn{6}{|c|}{ Posición Social Responsable Económico } \\
\hline Obrero & 6 & 11 & 10 & 27 & \\
\hline Sub-asalariado & 4 & 4 & 2 & 10 & $0.487 \dagger$ \\
\hline Comerciante & 1 & -- & -- & 1 & \\
\hline Total & 11 & 15 & 12 & 38 & \\
\hline \multicolumn{6}{|c|}{ Madre trabajadora } \\
\hline $\mathrm{Si}$ & 2 & 2 & 3 & 7 & $0.756 \dagger$ \\
\hline No responde & 9 & 13 & 9 & 31 & \\
\hline Total & 11 & 15 & 12 & 38 & \\
\hline \multicolumn{6}{|c|}{ Afiliación Mujer Sistema de Salud } \\
\hline Afiliadas & 10 & 15 & 12 & 37 & \\
\hline No afiliadas & 1 & -- & -- & 1 & \\
\hline Total & 11 & 15 & 12 & 38 & $0.377 \dagger$ \\
\hline \multicolumn{6}{|c|}{ Afiliación niño Sistema de Salud } \\
\hline $\mathrm{Si}$ & 10 & 13 & 12 & 25 & $0.616 \dagger$ \\
\hline \multicolumn{6}{|c|}{ Escolaridad } \\
\hline Primaria & 1 & -- & 3 & 4 & \\
\hline Secundaria & 5 & 8 & 7 & 20 & \\
\hline Técnic/tecnológ./univ. & 5 & 7 & 2 & 14 & \\
\hline Total & 11 & 15 & 12 & 38 & $0.572 \dagger$ \\
\hline \multicolumn{6}{|c|}{ Rango de ingreso familiar (SMLV) } \\
\hline Medio & 1 & 3 & -- & 4 & \\
\hline Uno & 1 & 9 & 5 & 15 & \\
\hline Dos y tres & 6 & 3 & 6 & 15 & \\
\hline Cuatro o más & -- & -- & 1 & 1 & \\
\hline No responde & -- & -- & 3 & 3 & \\
\hline Total & 11 & 15 & 12 & 38 & $0.015 \dagger$ \\
\hline \multicolumn{6}{|c|}{ Vivienda } \\
\hline Propia paga & 4 & 2 & 2 & 8 & \\
\hline Propia y pagándola & 1 & 1 & 2 & 4 & \\
\hline Alquilada & -- & 7 & 1 & 8 & \\
\hline Familia & 6 & 2 & 7 & 15 & \\
\hline Otra (invasión) & -- & 3 & -- & 3 & \\
\hline Total & 11 & 15 & 12 & 38 & $0.009 \dagger$ \\
\hline
\end{tabular}

SMLV: Salario Mínimo Legal Vigente. $\dagger$ Test exacto de Fisher.

Fuente: elaboración propia 
Algunos aspectos de los modos de vida de las familias que se constituyen en procesos limitantes o protectores se referencian a continuación, acompañados de fragmentos que recogen la voz de las mujeres entrevistadas o participantes de grupos de discusión.

\section{Dinámicas laborales de las mujeres}

Se observó una tendencia a la deslaboralización durante la etapa del embarazo, en unos casos de manera voluntaria, en otros por presión de la pareja o por discriminación.

... por eso las empresas no contratan mujeres embarazadas... porque para los jefes somos un gasto las mujeres embarazadas porque tienen que pagar pensión y salud. (Mujer, Bucaramanga, 27 años).

El otro caso es aquel en el que, a pesar de la voluntad de las mujeres de aportar ingresos al hogar, su familia extensa no permite que busquen empleo. Algunas lo toman como algo que las favorece en tanto les da el tiempo para cuidar del desarrollo biológico y emocional del recién nacido.

... mi esposo dijo, no amor, deje de trabajar y más bien se pone juiciosa con la revista de $* * *$ y se está en la casa y se cuida y va al médico.... entonces dejé de trabajar. (Mujer, Bucaramanga, 20 años).

Sin embargo, no en todos los casos esta restricción a tener un empleo es bien recibida, puesto que las mujeres sienten coartada su libertad de decidir, su independencia, su posibilidad de construir una historia laboral y de acceder al sistema de seguridad social. "Porque me gusta trabajar, no me gusta estar esperando a que él me quiera dar, no me gusta pedirle para mis hijas y yo quedarme sin hacer nada." (Mujer, Medellín, 29 años).

\section{Dinámicas de consumo de servicios de salud}

Se encontró la vinculación de la mujer a los programas de atención y protección integral que reciben durante el periodo prenatal y postnatal (en el caso de Chile) y la vinculación temprana de los niños al programa de crecimiento y desarrollo (Colombia). Estos programas tienen un acompañamiento educativo que promueve conocimientos y prácticas de cuidado de la salud en estas mujeres y sus hijos, por lo tanto, incentivan en mayor o menor medida, el derecho a la salud bucal y como parte de él, la demanda de la consulta odontológica. "Y esos talleres eran enfocados... Había un taller de dentista, que te explicaban todo lo que consistía con los dientes, con la comida, con los alimentos..." (Mujer, Santiago, 30 años).

Como procesos del consumo que limitan el derecho a la salud bucal se destacan las barreras geográficas relativas al servicio de transporte para llegar a los servicios de salud, en ciudades muy segmentadas en términos socioeconómicos. También se identifican barreras institucionales relacionadas con la afiliación, con una capacidad instalada insuficiente para cubrir la demanda de servicios odontológicos, los horarios de atención, la itinerancia del recurso humano de los centros de salud. 
Ángela María Franco Cortés/ Marco Antonio Cornejo Ovalle/ Iris

Lucía Espinoza Santander/ Clara Liliana Gallego Gómez/ et al.

Hay que madrugar mucho [para solicitar cita odontológica] porque reparten poquitos fichos. Y si usted llega y ya los repartieron, vuelva y madrugue. Allá es un horario corto [para la atención], de 7 de la mañana a las

3 de la tarde, lo que puedan atender en ese trayecto. (Participante grupo de discusión, Medellín).

Algunas mujeres relatan necesidades en salud bucal acumuladas por la falta de continuidad en los tratamientos. Es frecuente que a las mujeres embarazadas se les priorice para la primera cita odontológica, pero para el resto del tratamiento tienen las mismas limitaciones de la población general para acceder al servicio. Hallazgo que coincide con lo referido en la encuesta de modos de vida en la que se señala que el 50\% de las mujeres en Bucaramanga y el 58,3\% de las de Medellín, terminaron los tratamientos que les iniciaron en el embarazo, mientras que cerca del $78 \%$ de las mujeres en Santiago lograron culminarlos.

Estas necesidades acumuladas y no resueltas pueden llegar a complicarse durante el postparto con consecuencias desde el dolor y el sufrimiento hasta la pérdida de piezas dentales y la insatisfacción con el tratamiento, pues perciben que la atención que les ofrecen es de baja calidad e inoportuna.

Muy maluca, yo me siento muy mal. A veces me salen malos olores, todo el día me tengo que cepillar, me cepillo, pero no me hago con la seda dental porque me duele. (Mujer, Medellín, 30 años).

Se encuentra que para las mujeres, el postparto se convierte en una etapa que las invisibiliza en relación con la atención por lo que prima en su discurso una sensación de pérdida de los privilegios que tenían en el embarazo, lo que genera sentimientos de frustración e impotencia frente a sus posibilidades en el Sistema de Salud.

Hay más facilidades para las mujeres cuando están embarazadas, pero esos derechos se pierden... (Mujer, Bucaramanga, 22 años).

Yo no sé, pero uno en embarazo lo atienden más rápido. Mejor dicho, embaracémonos a cada rato. (Mujer, Medellín, 28 años).

Un hallazgo significativo fue que, pese a la legislación que protege a los niños menores de un año, las instituciones suelen recomendarles que lleven a sus hijos al primer control odontológico a partir de los dos o tres años. "Yo hablé y me dijeron que todavía no, que por ahí a los dos años o dos años y medio lo llevara a odontología." (Mujer, Bucaramanga, 24 años).

\section{Dinámicas socioculturales}

Lo que las participantes de las tres ciudades construyen en su cotidianidad y tienen en sus imaginarios como realidades posibles las condiciona para reconocer o no sus derechos en salud bucal y la de sus hijos y la posibilidad de exigirlos en el caso de ser vulnerados. Estas realidades se construyen por vivencias propias, por su participación en los programas sociales, por su 
interacción con las instituciones y con los profesionales que las atienden, por las experiencias de sus familiares cercanos, por la tradición oral transmitida a través de las generaciones, y por la influencia que el medio ejerce sobre sus convicciones, representaciones y experiencias. Destacan que la participación en los programas como el de crecimiento y desarrollo, FAMI o Chile Crece Contigo les permitió aprender a cuidar la boca de sus bebés. "Sí, porque uno aprende harto con lo que las parvularias enseñan (Mujer, Santiago, 28 años)".

También esta participación se convierte en una oportunidad para que las mujeres generen nuevas redes de apoyo, aprendan, compartan experiencias, resuelvan dificultades relacionadas con los cuidados del bebé, su interacción con el sistema de atención, los mecanismos que implementan para hacer efectivo su derecho a la salud. En resumen, estos programas se viven y se entienden como medio de empoderamiento de la mujer y son muy valorados por ellas.

Yo me salí de *** y ahí fue que empecé a tener todos esos problemas, que el mes de prueba, que no estaba afiliada, luego otra vez y así; ya la última, me tocó ponerme brava con la señora, decirle que yo tenía una niña y que si ella se enfermaba entonces quién me iba a responder y ella me dijo que sí señora, que pena y ya sí quedó afiliada... (Mujer, Bucaramanga, 18 años).

\section{Procesos determinantes del nivel singular}

\section{La situación de salud bucal de las mujeres en postparto (singular)}

Los resultados del examen bucal muestran que la experiencia de caries de las mujeres participantes del estudio es alta teniendo los valores más altos la ciudad de Bucaramanga, ver tabla 3. Con respecto a la condición periodontal de las madres, un alto porcentaje de ellas en Medellín y Bucaramanga presentaron sangrado y cálculos. En Santiago de Chile se observó alta presencia de sangrado y en algunas mujeres presencia de bolsas periodontales con pérdida de inserción leve. 
Ángela María Franco Cortés/ Marco Antonio Cornejo Ovalle/ Iris

Lucía Espinoza Santander/ Clara Liliana Gallego Gómez/ et al.

Tabla 3 Índice COP-D de las madres participantes, primer y segundo examen

\begin{tabular}{|l|l|c|c|c|c|c|c|}
\hline Ciudad & Examen & (n) & $\begin{array}{c}\overline{\mathrm{X}} \text { caries } \\
\text { NO } \\
\text { cavitada }\end{array}$ & $\begin{array}{c}\overline{\mathrm{X}} \text { caries } \\
\text { cavitada }\end{array}$ & $\begin{array}{c}\overline{\mathrm{X}} \\
\text { obturado }\end{array}$ & $\begin{array}{c}\overline{\mathrm{X}} \\
\text { perdidos }\end{array}$ & $\overline{\mathrm{X}}$ COP-D \\
\hline \multirow{2}{*}{ Medellín } & Primer & 12 & 0,6 & 0,5 & 4,0 & 0 & 5,1 \\
\cline { 2 - 8 } & Segundo & 8 & 0 & 0,1 & 3,2 & 0,1 & 3,5 \\
\hline \multirow{2}{*}{ Bucaramanga } & Primer & 15 & 4,4 & 3,2 & 5,5 & 0,1 & 13,2 \\
\cline { 2 - 9 } & Segundo & 14 & 4,4 & 4,5 & 6,1 & 0,4 & 15,5 \\
\hline \multirow{2}{*}{$\begin{array}{l}\text { Santiago de } \\
\text { Chile }\end{array}$} & Primer & 13 & 0,6 & 0,8 & 4,4 & 0,9 & 6,7 \\
\cline { 2 - 9 } & Segundo & 13 & 0,6 & 0,8 & 4,5 & 1,0 & 6,9 \\
\hline
\end{tabular}

Nota: segundo examen practicado 12 meses después. $\overline{\mathrm{X}}$ Promedio dientes.

Fuente: elaboración propia.

\section{Situación de salud bucal del niño}

En ninguna de las tres ciudades, en la primera visita se reportaron niños/as con experiencia de caries debido a que la gran mayoría de ellos, por la edad, aún no tenían dientes. Como era de esperarse para la segunda visita todos los niños tenían al menos uno o varios dientes, sin embargo, ninguno presentaba experiencia de caries.

\section{Discusión y conclusiones}

Los resultados de este estudio confirman que en las tres ciudades entran en juego procesos críticos, generales, particulares, relacionados con las dinámicas laborales, de consumo, socioculturales, y procesos singulares, que para este estudio fueron los relativos a la situación de salud bucal de ambos participantes, la mayoría de los cuales limitan el ejercicio efectivo de este derecho.

Procesos económico-sociales de reducción de la inversión y la protección social (educación, seguridad alimentaria, vivienda, salud, trabajo) de responsabilidad del Estado, soportados en una política de focalización en los grupos más pobres y vulnerables, principalmente en Colombia, aunque también en Chile; políticas focalizadas que en muchos casos no alcanzan a cubrir a nuestros participantes por provenir de familias que hacen parte de las capas pobres de la sociedad. 
Procesos políticos relativos a la existencia de políticas de salud y de organización del sistema sanitario desde una concepción de mercado y de intermediación financiera de los recursos públicos por parte de aseguradoras privadas, más interesadas en su propia rentabilidad que en la garantía del derecho fundamental a la salud. Esta decisión política sobre la organización del sistema de salud excluye de él a sectores amplios de la población. Lo anterior, que es la situación dominante, no desconoce que en ambos países existe una legislación y unas políticas sociales, y de salud particularmente, dirigidas a estos dos grupos que tratan de subsanar algunos vacíos propios del modelo de salud.

Procesos propios de las relaciones de género que ponen a estas mujeres y sus hijos en situación de vulnerabilidad, especialmente por los cambios en la vinculación laboral que se configuran en esta etapa. Todos estos procesos actúan como un sustrato estructural que perpetúa la pobreza y la inequidad impidiendo incluso que las políticas sociales sean lo suficientemente efectivas como para garantizar la vida saludable.

Cabe destacar que en el nivel estructural de las políticas de atención y en el particular del modelo de atención se registran algunas diferencias entre los dos países con ligeras ventajas para la sociedad chilena. Chile ha definido un modelo de garantías de derechos exigibles en atención odontológica para mujeres gestantes, y desde 2016 permite que puedan seguir su tratamiento ya no solo hasta el noveno mes postparto sino hasta 15 meses después de la primera consulta odontológica en etapa de embarazo.

La intermediación financiera en Colombia genera una ineficiencia social que deriva, entre otros, en la falta de control del Estado, la carencia de sistemas de información confiables, la incapacidad administrativa y financiera, la vulneración de derechos de los usuarios, la conversión de la salud en un bien de mercado, el deterioro en la calidad de la atención, la reducción de los recursos financieros por la burocracia administrativa y las utilidades en la intermediación y la manipulación política de los subsidios a la demanda (39).

Sin duda, los gobiernos de corte social que han existido en algunos periodos de la historia reciente de Chile marcaron una diferencia importante en cuanto a políticas sociales de más amplia cobertura y, por lo tanto, algunas diferencias en el ejercicio de los derechos. Por ejemplo, se identificó que la existencia del llamado permiso postnatal en este país hace posible que las mujeres en postparto puedan acceder a los cuidados y a la atención odontológica. En Colombia muchas mujeres no acceden a la licencia de maternidad por su desvinculación laboral en el embarazo, lo que produce una falla en materia de derecho a la salud y, por ende, a la salud bucal.

Los procesos del nivel particular, que se derivan de la posición social de las familias a las que pertenecen las mujeres y sus hijos, obreras y sub-asalariadas principalmente, tienen que ver con dinámicas laborales, de consumo, socioculturales y de capacidad organizativa y de empoderamiento de estos grupos sociales. 
Ángela María Franco Cortés/ Marco Antonio Cornejo Ovalle/ Iris

Lucía Espinoza Santander/ Clara Liliana Gallego Gómez/ et al.

Como parte de las dinámicas laborales se destacaron procesos como el del desempleo o la pérdida del empleo que las mujeres enfrentan durante el embarazo presionadas en muchas ocasiones por su pareja o por la familia. Estos procesos terminan siendo una amenaza para la salud bucal propia y la de sus hijos por cuanto el aseguramiento en salud -y las oportunidades de atención- está relacionado con el hecho de estar empleada y con la capacidad adquisitiva que proporcione el empleo.

En el caso de las dinámicas de consumo que limitan el derecho a la salud bucal, los procesos críticos identificados fueron aquellos que se producen en las instituciones de servicios, procesos que tienen que ver con las barreras geográficas, con la inapropiada organización de los horarios de atención, la baja capacidad instalada (servicios de segundo y tercer nivel casi inalcanzables), la itinerancia del recurso humano responsable de la atención, los costos adicionales que deben cubrir los mismos usuarios y la calidad de la atención.

En concordancia con lo anterior los trabajos de Cantarutti et al. (40) sobre gestantes, Gwengu (41) en el postparto y Lamarca et al. (42) en mujeres embarazadas y en postparto, concentran su atención en barreras que denominan "estructurales", como son el costo, las características del sistema y de los proveedores de la atención, la influencia del vecindario o el capital social como factores que determinan el acceso a la atención.

En el caso del proceso de consumo de atención odontológica de los niños, Franco-Cortés et al. (43) señalan que el desconocimiento de los derechos, la capacidad de pago y la organización de los servicios parecen influenciar el acceso a la atención odontológica. Mofidi (44) reporta que las dificultades para obtener una cita, la asistencia a la consulta asociada al estrés y los tiempos de espera, el desplazamiento, la interacción con el personal administrativo y de la atención y el sistema de vinculación emergen como aspectos que reducen la posibilidad de que los niños accedan a la consulta; refiere además que las barreras raciales y étnicas, así como las consecuencias psicológicas, tanto para el niño como para la persona que lo acompaña, inciden en la asistencia a la consulta.

Cardozo y Bernal (45) señalan que las mujeres durante el puerperio tienen su propia forma de cuidarse y en ello juegan un papel importante, además de la propia mujer, su familia y las mujeres con quienes comparte sus experiencias del cuidado, así como los profesionales y personal de salud que las atienden por lo que es fundamental ofrecer un cuidado culturalmente situado que tenga en cuenta el cuidado profesional pero también el cuidado popular aspecto que coincide con los hallazgos de este trabajo cuando se identifica la importancia de las experiencias educativas en relación con el cuidado bucal, como escenarios para el intercambio de saberes en un diálogo no necesariamente contradictorio, entre el modelo biomédico y el médico popular.

En cuanto a los procesos de orden singular, las condiciones bucales identificadas en las mujeres en postparto tanto en Bucaramanga y Medellín como en Santiago de Chile son comparables con 
otros reportes. Yllesca-Yllesca et al. (46), en Perú, señalan que cuando se alude a la condición periodontal el $100 \%$ de las mujeres que lactaban sus hijos registraron sangrado al sondaje y el $33 \%$ bolsas periodontales. Cartes-Velásquez et al. (47) refieren que al evaluar el estado de salud bucal en madres beneficiarias del sistema Chile Crece Contigo se reportó que la media del COPD fue de $14,6 \pm 5,52$, alcanzando valores mayores en mujeres mayores de 20 años $(15,8 \pm 5,2)$, y multíparas $(16,7 \pm 5,0)$.

En cuanto a la condición bucal de los infantes, en Colombia, el ENSAB IV (2014) (48) encontró que un $5,9 \%$ de los niños de un año tenía caries dental cavitada; y un $26,9 \%$ caries en etapas precavitadas (mancha blanca). En Santiago de Chile, para el año de 2015 Belmar-Rebolledo (49) identificó una prevalencia de caries dental en niños de 2 y 3 años (ICDAS II 2-6) del 46,2\%, con un $23,4 \%$ de lesiones cavitadas en dentina (ICDASII 5 y 6 ). A pesar de que en general los estudios en niños menores de 6 años no son muy alentadores se puede afirmar que en los grupos de niños evaluados en esta investigación el panorama con relación a la caries es bueno. Sin embargo, el bajo acceso a la atención preventiva oportuna podría llegar a comprometer la salud bucal de los niños.

En síntesis, bajo una perspectiva de determinación social se corrobora que el derecho a la salud bucal de las mujeres en periodo de postparto y de sus recién nacidos está amenazado por procesos limitantes del nivel general (económicos, políticos y de género) que subsume a otros procesos del nivel particular (dinámicas laborales, de consumo y socioculturales) y estos, a su vez, a otros del nivel singular (condiciones bucales de las mujeres y sus hijos menores de un año). Estos procesos desfavorecedores disminuyen la efectividad de algunos pocos procesos que se comportan como favorecedores de tal derecho.

A manera de conclusión general sobre el enfoque puede afirmarse que en esta investigación se identificaron fortalezas relativas al abordaje epistemológico y metodológico, ya que el enfoque de la determinación social de la salud y la integración de abordajes cualitativos y cuantitativos sirvió para obligar a los investigadores a trascender la mirada tradicional de la condición bucal en las mujeres y sus hijos y los "factores" asociados, en tanto requirió observar otros niveles de la realidad de estos sujetos que determinan la situación objetiva encontrada.

Bajo el concepto de derecho a la salud bucal y de procesos asociados a su realización se logró superar la mirada biologicista y asistencialista con la que normalmente se abordan estos estudios. Este derecho, como un componente del derecho a la salud, no sólo se asocia a cuidados sino que también se relaciona con determinantes sociales e inequidad (50); por lo tanto, se aporta una visión renovada a la comprensión del tema.

\section{Consideraciones éticas}

Esta investigación se rigió en Colombia por la Resolución 08430 de 1993 y en Chile por la Ley No. 20.584. La investigación fue aprobada por los Comités de ética de las instituciones involucradas. 
Ángela María Franco Cortés/ Marco Antonio Cornejo Ovalle/ Iris

Lucía Espinoza Santander/ Clara Liliana Gallego Gómez/ et al.

\section{Contribución de los autores}

Todos los autores contribuyeron en la elaboración del estudio, asimismo leyeron y aprobaron la versión final del manuscrito remitido a la Revista.

\section{Conflictos de interés}

Los autores declaran no tener ningún tipo de conflicto de interés.

\section{Referencias}

1. Corchuelo-Ojeda J. A literature review on social and economic factors related to access to dentalcare for pregnant women. J Dent. 2013;1(1):24-34. https://doi.org/10.12974/2311-8695.2013.01.01.4

2. Saddki N, Yusoff A, Hwang YL. Factors associated with dental visit and barriers to utilisation of oral health care services in a sample of antenatal mothers in Hospital Universiti Sains Malaysia. BMC Public Health. 2010;10:75. https://doi.org/10.1186/1471-2458-10-75

3. Lamarca GA, do C Leal M, Sheiham A, Vettore MV. The association of neighborhood and individual social capital with consistent self-rated health: A longitudinal study in Brazilian pregnant and postpartum women. BMC Pregnancy Childbirth. 2013;13:1. https://doi.org/10.1186/1471-2393-13-1

4. Corchuelo-Ojeda J, Pérez GJ. Socioeconomic determinants of dental care during pregnancy in Cali, Colombia. Cad Saúde Pública. 2014;30(10):2209-2218. https://doi.org/10.1590/0102-311X0015241 3

5. Concha-Sánchez SC, Morales C. Determinación social de la atención odontológica de las mujeres embarazadas de tres localidades de Bogotá, Tesis Doctoral. Bogotá: Universidad Nacional de Colombia; 2015.

6. Kulikova NG, Omeltchuk NN, Zalenskiy VA, Tkachenko AS. The organization of medical stomatological care of women in post-natal period. Probl Sotsialnoi Gig Zdravookhranenniiai Istor Med. 2014;3:34-36. https://doi.org/10.32687/0869-866x-2019-27-2-204-209

7. Lamarca, GA, Leal Mdo C, Leao AT, Sheiham A, Vettore MV. Oral health related quality of life in pregnant and post partum women in two social network domains; predominantly home-based and work-based networks. Health Qual Life Outcomes. 2012;10:5. https://doi.org/10.1186/1477-7525-1 $0-5$

8. Riedy CA, Weinstein P, Milgrom P, Bruss M. An ethnographic study for understanding children's oral health in a multicultural community. Int Dent J. 2001;51(4):305-312. https://doi.org/10.1002/j.1875 -595X.2001.tb00843.x

9. Plutzer K, Spencer AJ. Efficacy of an oral health promotion. Intervention in the prevention of early childhood caries. Community Dent Oral Epidemiol. 2008;36(4):335-346. https://doi.org/10.1111/j.1 600-0528.2007.00414.x 
Procesos de determinación del derecho a la salud bucal de mujeres en post-

parto y sus hijos. Bucaramanga y Medellín (Colombia), Santiago (Chile), 2017

10. Hernández M. Neoliberalismo en salud: desarrollos, supuestos y alternativas. En: Restrepo D (ed). La falacia neoliberal: crítica y alternativas. Bogotá: Universidad Nacional de Colombia; 2003. p.347-361.

11. Constitución Política de Colombia. Bogotá DC: Presidencia de la República; 1991.

12. Cornejo-Ovalle M, Brignardello-Petersen R, Pérez G. Pay-for-performance andefficiency in primary oral health care practices in Chile. Rev Clín Periodoncia Implantol Rehabil Oral. 2015;8(1):60-66. h ttps://doi.org/10.1016/j.piro.2015.02.011

13. Breilh J. Epidemiología crítica: ciencia emancipadora e interculturalidad. Buenos Aires: Lugar Editorial; 2003.

14. Breilh J. Las tres "S" de la determinación de la vida: 10 tesis hacia una visión crítica de la determinación social de la vida y la salud. En: Passos-Nogueira R (ed.) Determinação Social da Saúde e Reforma Sanitária. Rio de Janeiro: Cebes; 2010.

15. Gallego CL, Franco-Cortés AM. Procesos determinantes del acceso a la atención odontológica de niños y niñas durante la primera infancia. Medellín, 2016. Tesis Maestria en Ciencias Odontológicas. Medellín: Universidad de Antioquia; 2017.

16. Quintero CA, Robles DP, Vásquez A, Delgado O, Franco AM. Barreras de acceso a la atención odontológica durante la primera infancia. Medellín, 2007. Rev Fac Odontol Univ Antioq. 2014;25(2):325-341. http://www.scielo.org.co/pdf/rfoua/v25n2/v25n2a07.pdf

17. Concha-Sánchez SC. El proceso salud-enfermedad-atención bucal de la gestante: una visión de las mujeres con base en la determinación social de la salud. Rev Fac Med. 2013;61(3):275-291. http:// www.scielo.org.co/pdf/rfmun/v61n3/v61n3a08.pdf

18. Breilh J. Nuevos conceptos y técnicas de investigación. Guía pedagógica para un taller de metodología (epidemiología del trabajo). Quito: Centro de Estudios y Asesoría en Salud; 1979.

19. Breilh J. Latin American critical (Social) epidemiology: New settings for an old dream. Int J Epidemiol. 2008;37(4):745-750. https://doi.org/10.1093/ije/dyn135

20. Breilh J. Epidemiología económica, política y salud: bases estructurales de la determinación social de la salud. Quito: Corporación Editora Nacional - Universidad Andina Simón Bolívar; 2010.

21. Breilh J. La determinación social de la salud como herramienta de transformación hacia una nueva salud pública (salud colectiva). Rev Fac Nac Salud Pública. 2013;31(Supl 1): S13-S27. http://www.sciel o.org.co/pdf/rfnsp/v31s1/v31s1a02.pdf

22. Bogdan R, Taylor S. Introducción a los métodos cualitativos de investigación. Barcelona: Paidós; 1986.

23. Departamento Administrativo Nacional de Estadística (DANE). Boletín técnico: pobreza monetaria y multidimensional en Colombia 2015. Bogotá: DANE, 2016. https://www.dane.gov.co/files/investig aciones/condiciones_vida/pobreza/bol_pobreza_15_.pdf

24. Departamento Administrativo Nacional de Estadística (DANE). Boletín técnico: pobreza monetaria y multidimensional en Colombia 2016. Bogotá: DANE; 2017. http://www.dane.gov.co/files/investiga ciones/condiciones_vida/pobreza/bol_pobreza_16.pdf 
Ángela María Franco Cortés/ Marco Antonio Cornejo Ovalle/ Iris

Lucía Espinoza Santander/ Clara Liliana Gallego Gómez/ et al.

25. PNUD. Estudio del PNUD revela seis nudos de reproducción $\mathrm{y}$ cambio de la desigualdad en Chile. PNUD Chile; 14 de junio de 2017. Disponible: http://www.cl.undp.org/content/chile/es/home/presscenter/pressreleases/2017/06/14/est udio-del-pnud-revela-seis-nudos-de-reproducci-n-y-cambio-de-la-desigualdad-en-chile.html

26. OECD. Income inequality and poverty. OECD. www.oecd.org/social/inequality-and-poverty.htm.

27. Vélez M. La salud en Colombia: pasado, presente y futuro de un sistema en crisis. Bogota: Penguin Random House; 2016.

28. Muñoz J. Concentración de la tierra un debate que viene. Diario Uchile. 18 de octubre 2014. Sección Medio Ambiente/Política. http://radio.uchile.cl/2014/10/18/concentracion-de-la-tierra-un-debate-qu e-viene/

29. Quiroga Y, Ensigna J. Chile en la concertación, 1990-2010. Una mirada crítica, balance y perspectivas. Santiago de Chile: Friedrich Ebert Stifung; 2010.

30. Universidad del Desarrollo. Centro de Estudios y Economía y Negocios. Ranking de grupos económicos RGE. Concepción (Chile): Universidad del Desarrollo; 2016. http://www.udd.cl/wp-content/upload s/2016/06/RGE-Diciembre-2015-.pdf

31. Bogliacino F, Chavarro W, García JG, Giraldo C, Junca G, et al. Plan Nacional de Desarrollo 2014-2018: siempre el mismo país. Bogotá: Universidad Nacional de Colombia; 2016. http://www.cid.unal.edu .co/cidnews/archivos/coleccion-controversias-sobre-desarrollo.pdf

32. Saavedra E. El modelo económico-político de Chile: desarrollo institucional en la encrucijada. Economía y Política. 2014;1(1):117-148. economiaypolitica.cl/index.php/eyp/article/view/7/4

33. Hernández-Vásquez A, Azañedo D, Díaz-Seijas D, Bendezú-Quispe G, Arroyo-Hernández H, et al. Acceso a los servicios de salud en menores de doce años en Perú, 2014. Salud Colect. 2016;12(3):429-441. https://doi.org/10.18294/sc.2016.912

34. Chile. Superintendencia de Salud. Evaluación de la implementación de la reforma de salud en el sistema Isapres y su impacto en los beneficiarios. Santiago de Chile: Superintendencia de Salud; 2007. http: //www.supersalud.gob.cl/documentacion/666/articles-4203 recurso_1.pdf

35. Colombia. Ley 1257 del 4 diciembre de 2008. Por la cual se dictan normas de sensibilización, prevención y sanción de formas de violencia y discriminación contra las mujeres, se reforman los Códigos Penal, de Procedimiento Penal, la Ley 294 de 1996 y se dictan otras disposiciones. Bogotá: Ministerio de Salud y Protección Social; 2008.

36. Andrade JA, Alvis L, Jiménez LK, Redondo MP, Rodríguez L. La vulnerabilidad de la mujer en la guera y su papel en el postconflicto. Ágora USB. 2017;17(1):290-308. https://doi.org/10.21500/16 578031.2827

37. Baeza-Reyes AV. Mujeres en la arena política: representaciones sociales de las candidatas parlamentarias en Chile de 2013. Tesis para el título profesional de socióloga. Santiago de Chile: Universidad de Chile; 2014. 
Procesos de determinación del derecho a la salud bucal de mujeres en post-

parto y sus hijos. Bucaramanga y Medellín (Colombia), Santiago (Chile), 2017

38. Ministerio de la Mujer y Equidad de Género. Chile. Violencia contra las mujeres. Chile; 2017. http://w ww.minmujeryeg.cl/sernameg/programas/violencia-contra-las-mujeres/

39. Granados R, Gómez MC. La reforma de los sistemas de salud en Chile y Colombia. Resultados y Balance. Rev Salud Pública. 2000;2(2):97-120. https://revistas.unal.edu.co/index.php/revsaludpubli ca/article/view/18863

40. Cantarutti C, Veliz C, Pardo A, Ortúzar I, Jordan V, et al. ¿Cuáles son las barreras de acceso a la atención odontológica de las mujeres embarazadas? ARS Médica. 2017;42(2):50-54. https://doi.org/10.1156 5/arsmed.v42i2.890

41. Gwengu P. Determinants of utilization of oral health services by postnatal mothers in Winterveldt, Gauteng Province, South Africa. J Oral Hyg Health. 2017;5(1):222. https://doi.org/10.4172/2332-0 702.1000222

42. Lamarca GA, Leal Mdo C, Leao AT, Sheiham A, Vettore MV. The different roles of neighbourhood and individual social capital on oral health-related quality of life during pregnancy and postpartum: A multilevel analysis. Community Dent Oral Epidemiol. 2014;42(2):139-150. Epub 2013 Jul 22. h ttps://doi.org/10.1111/cdoe.12062

43. Franco-Cortes AM, Ramírez Puerta S, Escobar-Paucar G, Isaac-Millán M, Londoño-Marín PA. Barrerras de acceso a los servicios odontológicos de niños y niñas menores de 6 años pertenecientes a familias desplazadas. Rev CES Odont. 2010;23(2):41-48.

44. Mofidi M, Rozier G, King RS. Problem with access to dental care for Medicaid-Insured children: What caregivers think. Am J Public Health. 2002;92(1):53-58. https://doi.org/10.2105/ajph.92.1.53

45. Cardozo SL. Bernal MC. Adolescentes en puerperio y su práctica de cuidado. Rev Av Enferm. 2009;27(2):82-91. https://revistas.unal.edu.co/index.php/avenferm/article/view/12970

46. Yllesca-Ylleca I, Manrique-Chávez JE, Chávez-Reátegui BC. Características epidemiológicas de la enfermedad periodontal e higiene oral en las mujeres en etapa de embarazo y lactancia. Rev Estomatol Herediana. 2015;25(4):255-261. http://www.scielo.org.pe/pdf/reh/v25n4/a02v25n4.pdf

47. Cartes, Velásquez R, Mardones S, Paredes C. Conocimientos y estado de salud bucal en madres beneficiarias del sistema Chile Crece Contigo. Rev Chil Salud Pública. 2009;13(3):136-142. https:/ /doi.org/10.5354/0719-5281.2010.524

48. IV Estudio Nacional de Salud Bucal-ENSAB IV- Situación en Salud Bucal. Ministerio de Salud. Colombia; 2014. https://www.minsalud.gov.co/sites/rid/Lists/BibliotecaDigital/RIDE/VS/PP/ENSA B-IV-Situacion-Bucal-Actual.pdf

49. Belmar-Rebolledo BC. Estudio de prevalencia, índice significante, severidad de caries y distribución de lesiones por diente y superficie, en niños de 2 y 3 años pertenecientes a jardines infantiles integra de la región metropolitana. Repositorio academico Universidad de Chile. Trabajo de grado; 2015. http://repositorio.uchile.cl/bitstream/handle/2250/137559/Estudio-de-prevalencia\%2c-\%C3\% ADndice-significante $\% 2 \mathrm{c}$-severidad-de-caries.pdf?sequence $=1 \&$ isAllowed $=\mathrm{y}$

50. Rueda G, Albuquerque A. El derecho a la salud bucal en la declaración de Liverpool. Rev. Bioét. 2017;25(2):224-233. https://doi.org/10.1590/1983-80422017252182 


\section{Ángela María Franco Cortés/ Marco Antonio Cornejo Ovalle/ Iris}

Lucía Espinoza Santander/ Clara Liliana Gallego Gómez/ et al.

\section{Notas}

1 Reconociendo que tanto los niños como las niñas son sujetos de derecho, aclaramos que en este estudio la palabra "niños" los incluye a ambos. 